\title{
Erratum
}

\section{Amino Acid Replacement in the Protein S5 from a Spectinomycin Resistant Mutant of Bacillus subtilis}

T. Itoh

Molec. gen. Genet. 144, 39-42 (1976)

On page 39 fourth line should read:

from it. The purified S5 proteins were digested with

T. Itoh 